\title{
The use of small-bore wire-guided chest drains for the management of feline pyothorax: A retrospective case series
}

\author{
Sara Del Magno ${ }^{1}$, Armando Foglia ${ }^{1}$, Linda Golinelli ${ }^{1}$, Deborah De Bastiani ${ }^{2}$, Veronica Cola ${ }^{1}$, Luciano Pisoni ${ }^{1 *}$, \\ Lisa Grassato ${ }^{3}$, Marco Pelizzola ${ }^{4}$, Roberta Troia ${ }^{1}$ and Massimo Giunti ${ }^{1}$ \\ ${ }^{1}$ Department of Veterinary Medical Sciences, Alma Mater Studiorum, University of Bologna, Ozzano dell'Emilia, Italy \\ ${ }^{2}$ Clinica Veterinaria Gran Sasso, Milano, Italy \\ ${ }^{3}$ Fitzpatrick Referrals -Orthopaedics \& Neurology, Surrey, UK \\ ${ }^{4}$ San Marco Veterinary Clinic, Veggiano, Italy
}

\begin{abstract}
Background: Pyothorax in cats is routinely managed, at least initially, with thoracic tube placement associated with systemic antimicrobial administration. Traditionally, large-bore trocar-type thoracostomy tubes have preferentially been used for the drainage of thick material from the pleural space. In recent years, the use of small-bore wire-guided thoracic drains has increased in both small animals and in humans. Few studies have highlighted the efficacy of smallbore wire-guided thoracostomy tubes.

Aim: The purpose of this study was to describe the use of small-bore wire-guided thoracostomy tubes in feline pyothorax in terms of efficacy, safety, and outcome.

Methods: Cats with pyothorax managed with small-bore thoracostomy tubes (SBTTs) (2015-2018) were retrospectively studied. The number of drains inserted, the need for anesthesia and analgesia for chest tube placement and maintenance, and related major and minor complications were reviewed. Clinical data, diagnostic results, treatment, and outcome were recorded.

Results: Ten cats were enrolled. Thoracostomy tube placement was unilateral in $7 / 10$ cats, despite the presence of bilateral effusion in 9/10 cats, and required sedation (8/10) or anesthesia $(2 / 10)$. Three cats experienced minor complications during the chest tube insertion, including self-limiting pneumothorax (1/3) and malpositioning (2/3). One cat had a major complication (non-functional malposition) requiring reposition of the drain. Pain management was adequately achieved using opioids (8/10) or opioids plus nonsteroidal anti-inflammatory drugs (2/10). Partial chest tube occlusion occurred in three cases and it was resolved with lavage. In one case, the occlusion was complete, requiring drain removal. Three out of 10 cats were treated medically, combining thoracostomy tubes and antibiotics, while $7 / 10$ cats underwent surgery. All the cats survived.

Conclusion: SBTTs represent a safe and effective option for the initial management of feline pyothorax. In fact, mainly minor complications were reported during insertion and usage. The SBTTs were well tolerated by the cats with a satisfactory performance in terms of exudate drainage in most cases. The combined use of a small-bore thoracostomy drain together with the common practice of surgical treatment might have resulted in the successful management of the cases presented.
\end{abstract}

Keywords: Cat, Pyothorax, Thoracic empyema, Thoracic surgery, Thoracostomy drain.

\section{Introduction}

Pyothorax represents the accumulation of septic purulent exudate within the pleural space (Barrs et al., 2005; Stillion and Letendre, 2015). Numerous mechanisms have been considered as possible etiologies of pyothorax in cats. Although the literature regarding the predominant cause of feline pyothorax is controversial, parapneumonic infection spread from an adjacent intrathoracic structure, penetrating thoracic wounds, rupture of pulmonary abscess, migration of plant foreign bodies from the lung or the esophagus, and the perioperative aspiration of oropharyngeal flora can all be considered to be common potential reasons for the onset of pyothorax. However, the inciting insult remains undetermined in many cases as it does in dogs (Barrs et al., 2005; MacPhail, 2007; Beatty and Barrs, 2010; Epstein, 2014; Stillion and Letendre, 2015; Cola et al., 2019).

Medical and surgical options have both been proposed for the management of feline pyothorax; however, to date, there is no consensus regarding the treatment of choice, and precise indications are lacking in the veterinary literature. Medical treatment is usually started as a first attempt, while surgical management is generally recommended in the presence of mediastinal or pulmonary lesions detected by diagnostic imaging, or in cases where medical therapy fails (Demetriou et al., 2002; 
Waddell et al., 2002; MacPhail, 2007; Barrs and Beatty, 2009a; Stillion and Letendre, 2015).

Thoracocentesis is the first procedure carried out on both human and veterinary patients in order to obtain diagnostic samples and achieve initial stabilization (Tillison, 1997; MacPhail, 2007; Sigrist, 2015); however, it is rarely sufficient as the sole treatment for pyothorax (Barrs and Beatty, 2009b). Thoracocentesis is usually followed by thoracic tube placement associated with systemic antimicrobial administration (Tillison, 1997; MacPhail, 2007; Sigrist, 2015; Sherman et al., 2020).

Thoracostomy drains are divided into large-bore thoracostomy tubes (LBTTs) (14-16 Fr for cats and 24-26 Fr for dogs) (Drobatz et al., 2018) and small-bore thoracostomy tubes (SBTTs) (10-14 Ga) (Valtolina and Adamantos, 2009; Fetzer et al., 2017). LBTTs have a trocar to allow their insertion, and they have traditionally been used for the drainage of dense and thick material from the pleural space (Sigrist, 2015; Filosso et al., 2016; Hallifax et al., 2017). However, general anesthesia is usually required for their insertion, and many complications could occur during or after insertion in cats, such as laceration of the intrathoracic organs, hemorrhage, arrhythmias, and pain and discomfort (Demetriou et al., 2002; Waddell et al., 2002; Barrs et al., 2005; Valtolina and Adamantos, 2009).

In recent years, the use of SBTTs, introduced using the modified Seldinger technique, has increased in popularity in both small animals and humans (Valtolina and Adamantos, 2009; Hooper et al., 2015a; Stillion and Letendre, 2015). The advantages reported with the use of SBTTs include safe insertion with minimal sedation, a low complication rate, and elevated patient comfort during permanence (Valtolina and Adamantos, 2009). Recently, few studies have highlighted the efficacy of SBTTs in managing pleural space diseases, including pyothorax in dogs and cats (Barrs et al., 2005; Valtolina and Adamantos, 2009; Sigrist, 2015; Fetzer et al., 2017; Sherman et al., 2020).

The purpose of this study was to retrospectively evaluate the effectiveness of SBTTs in the initial management of feline pyothorax in terms of management, complications, and patient outcome.

\section{Materials and Methods}

The medical software program of the Veterinary University Hospital (VUH) of the University of Bologna in Italy was searched to find cats with pyothorax in which an SBTT (MILA International Inc., Florence, KY) had been inserted as the initial treatment from 2015 to 2018. The cases enrolled were part of a population referred for a first medical consultation to the VUH and were included in the study if a definitive diagnosis of pyothorax was obtained by means of cytology and/or bacterial culture of the pleural fluid sampled by thoracocentesis (MacPhail, 2007; Barrs and Beatty, 2009a).
The following information was retrieved and analyzed from the electronic reports for all the cats enrolled: age, sex, breed, history, clinical signs, initial stabilization treatment, complete blood analyses (blood gas analysis, complete blood count, chemistry, blood clotting tests), thoracic radiography, thoracic ultrasonography, cytology, and aerobic/anaerobic bacterial culture of the pleural fluid and antibacterials administered.

The sedation or anesthesia carried out for the thoracic tube insertion, unilateral or bilateral placement, complications during the thoracic drain insertion and use, length of stay of the thoracic tube, pain medications, and the requirement for supplemental surgical exploration and outcome, were also recorded. The SBTTs (MILA International Inc., $14 \mathrm{Ga}, 2-\mathrm{mm}$ diameter) (Fig. 1) were inserted using the modified Seldinger technique (Valtolina and Adamantos, 2009). A unilateral drain was initially applied, even in the case of bilateral effusion. In these situations, the drain was inserted in the site where the effusion was more abundant. In cases of bilateral effusion, the contralateral side was monitored using ultrasound examination and, if the amount of fluid persisted or increased, a second drain was placed. Intermittent suction and thoracic lavages using $20 \mathrm{ml}$ of warm sterile saline solution for each drain were carried out every 6-8 hours on the first day and adjusted according to the amount of fluid removed. Daily monitoring of the pleural effusion was carried out by serial ultrasonographic evaluation in order to assess the efficacy of intermittent aspirations and evaluate possible residual fluid in the pleural space.

Pain management was provided using opioids at the discretion of the attending clinician on duty in the intensive care unit.

Surgical treatment was advised and carried out when medical therapy failed (persistent production of septic purulent exudate after the first 3-5 days following

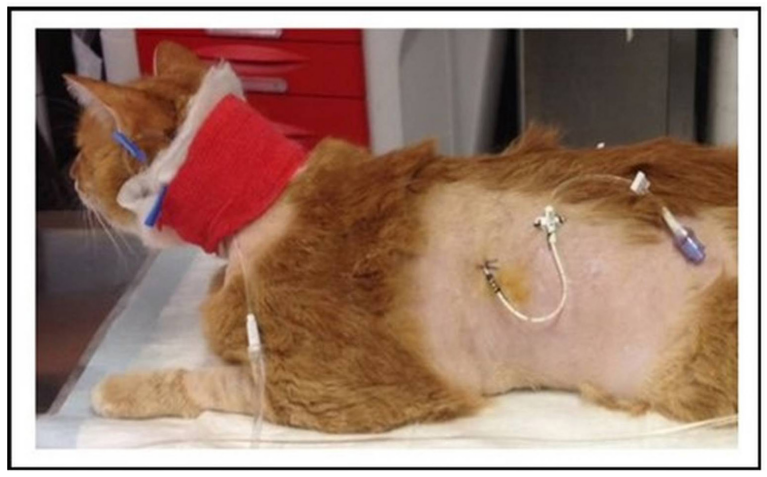

Fig. 1. Small-bore thoracostomy tube placed in a cat (Cat n.10, lateral view). Note the small-bore thoracostomy (14 $\mathrm{Ga}$ ) tube inserted in the left hemithorax in a cat with bilateral effusion, after removal of the thoracic bandage. The cat also had a central venous catheter in place covered by a neck bandage. 
SBTT insertion), or in the presence of mediastinal or pulmonary lesions identified on the diagnostic imaging. Complications were considered major if they were lifethreatening or if they required surgical intervention for their resolution, and minor in the other cases (e.g., malpositioning of SBTT).

\section{Statistical analysis}

The data were assessed for normality using the D'Agostino-Pearson test and the descriptive statistics were calculated as appropriate. Since most variables were not normally distributed, the data were expressed as median and range.

\section{Ethical approval}

The cats included in the study were client-owned cats, and owners signed an information consent form upon admission. No experiments were conducted on the animals.

\section{Results}

Ten cats with a diagnosis of pyothorax fulfilled the inclusion criteria and were enrolled in the study. Three cats were spayed females, two were intact females, and the remaining five were castrated males. Eight out of the 10 cases were domestic short-haired cats, 1 was a Persian cat and, 1 was a Bengal cat. The median age was 9 years (range: $0.8-11$ years) and the median body weight was $3.9 \mathrm{~kg}$ (range: $1.7-6 \mathrm{~kg}$ ).

The main historical data and clinical signs reported upon admission to the hospital were dyspnea with the presence of a restrictive respiratory pattern (9/10 cats), lethargy (6/10), anorexia (6/10), and abnormal lung sounds on thoracic auscultation (6/10) (Table 1).

The most common laboratory abnormalities observed were neutrophilic leukocytosis (6/10 cats) with a left shift, the presence of toxic neutrophil changes, decreased albumin (10/10), increased serum levels of aspartate aminotransferase (8/10), and a decreased concentration of total protein $(5 / 10)$ and serum acid phosphatase (7/10). Serum amyloid A concentration was available in $7 / 10$ cats and was elevated in all the patients. Prothrombin time and partial thromboplastin activation time were elevated in $7 / 10$ and $3 / 10$ cats, respectively (Tables $2-4$ ).

The initial thoracic ultrasound evaluation showed the presence of unilateral effusion in 1/10 cats and bilateral effusion in $9 / 10$ cats. Cytological evaluation of the pleural fluid collected by means of thoracocentesis was available for all the cats enrolled. Degenerated neutrophils (10/10 cats), monocytes (9/10 cats), and intracellular and/or extracellular bacteria were identified in all the cats. The results of the bacterial culture were available and the most common bacteria isolated was Actinomyces spp. (7/10 cats); other isolated pathogens were anerobic polymicrobial infection, Escherichia coli, Corynebacterium spp., Pasteurella spp., and Streptococcus spp. (Table 1).

Antimicrobial treatment included the administration of at least one of the following antibiotics: ampicillin- sulbactam (Unasyn®, Pfizer Inc, $20 \mathrm{mg} / \mathrm{kg}$ IV or IM every 12 hours) in $9 / 10$ cats, marbofloxacin (Marbocyl@, Vetoquinol, $3 \mathrm{mg} / \mathrm{kg}$ IV or IM daily) in $2 / 10$ cats, piperacillin-tazobactam (Tazocin $®$, Sandoz, $50 \mathrm{mg} / \mathrm{kg}$ IV or IM every 6 hours) in 1/10 cats, and ceftazidime (Ceftazidima®), Vitalis, $30 \mathrm{mg} / \mathrm{kg}$ IV every 8 hours) in 1/10 cats (Table 1 ). The median duration of the antimicrobial therapy was 29 days (range: 10-65 days), according to the clinical and diagnostic findings during the follow-up examinations and the clinician preference.

\section{Thoracic drain insertion}

The SBTT was inserted unilaterally in $7 / 10$ cases and bilaterally in the remaining $3 / 10$ cats (Table 1 ). In two cats, the surgeon on duty decided to perform a bilateral placement because after the positioning of the first thoracostomy tube, moderate/severe pleural effusion was still present on the contralateral side. In one of the three cats with bilateral thoracic tubes, the second thoracic drain was inserted after the first day due to the persistence of dyspnea and moderate effusion in the contralateral pleural space. Eight out of the 10 cats required sedation for the SBTT insertion, while general anesthesia was given to two patients (one cat had bilateral placement during the same procedure).

Thoracic radiographs were carried out in all the patients to confirm the correct placement of the thoracic tube, and pulmonary abnormalities were identified in $6 / 10$ cats, including atelectasis and suspected pulmonary lesions.

Three out of the 10 cats experienced minor complications during insertion and one cat had a major complication. The minor complications included malpositioning of the tube that was still functional $(n=2)$ and mild selflimiting pneumothorax $(n=1)$. The major complication consisted of malposition of the thoracic tube requiring repositioning via fluoroscopy (Table 1).

\section{Thoracic drain management}

Analgesia included the administration of buprenorphine (Buprenodale $\AA$, Dechra Ltd, $10-15 \mu \mathrm{g} / \mathrm{kg}$ IV or IM every 8 hours) in $9 / 10$ cats, meloxicam (Metacam $\AA$, Boehringer Ingelheim Div. Veter, $0.05 \mathrm{mg} / \mathrm{kg}$ PO daily) in $2 / 10$ cats, and methadone (Semfortan $\AA$, Eurovet Animal Health B.V., $0.1-0.2 \mathrm{mg} / \mathrm{kg}$ IV or IM every 4 hours) in $1 / 10$ cats.

Once the SBTT was in place, the complication most encountered was thoracostomy tube occlusion. Partial and recurrent occlusions occurred in 4/10 cases, which were usually resolved by lavage of the tube. In one out of these four cases, the thoracic tube occlusion was complete, necessitating the removal of the drain, and this was considered a major complication (Table 1). The drains were left in place for a median time of 5 days (range: $1-13$ days). The median amount of fluid aspirated was $6.4 \mathrm{ml} / \mathrm{kg} /$ day (range: $1.2-21.8 \mathrm{ml} / \mathrm{kg}$ /day).

\section{Outcome}

No death was registered, and all the cats survived to discharge, with a positive outcome. Three out of the 


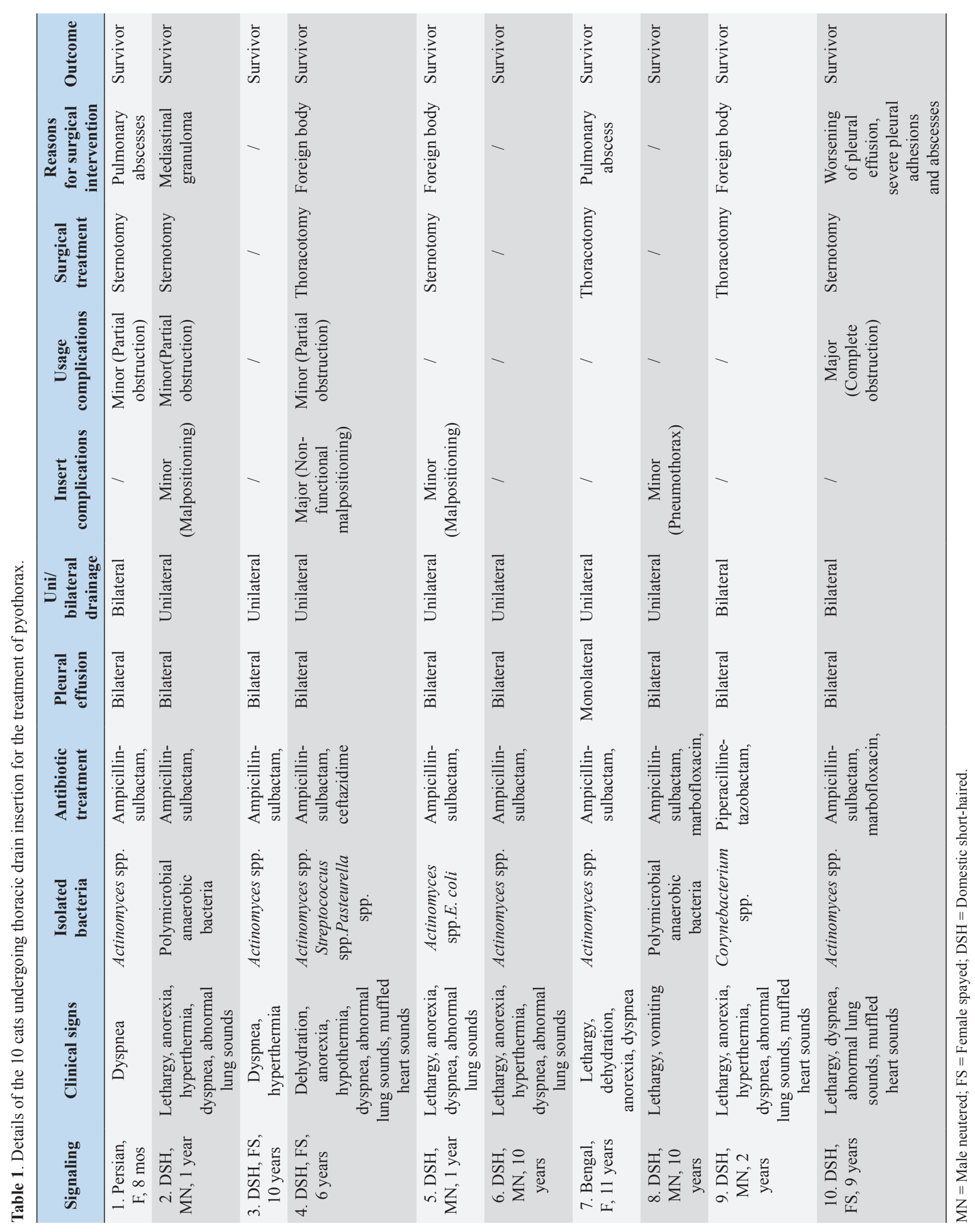


Table 2. Hematology at presentation of the 10 cats enrolled in the study.

\begin{tabular}{|c|c|c|c|c|c|}
\hline & Variable & Unit & $\begin{array}{l}\text { Median } \\
\text { value }\end{array}$ & $\begin{array}{c}\text { Range } \\
\text { (min-max) }\end{array}$ & Reference interval \\
\hline \multirow{4}{*}{$\mathrm{H}$} & Hemoglobin & $\mathrm{gr} \%$ & 12.1 & $7.4-13.4$ & $10.0-16.0$ \\
\hline & Hematocrit value & $\%$ & 35.3 & $21.7-39.7$ & $32.0-48.0$ \\
\hline & $\mathrm{RCBs}$ & $\mathrm{mm}^{3}$ & $7,800,000$ & $4,320,000-9,120,000$ & $7,000,000-11,000,000$ \\
\hline & $\mathrm{MCV}$ & $\mathrm{fl}$ & 44.3 & $40.9-50.2$ & $36.0-55.0$ \\
\hline \multirow{2}{*}{$\begin{array}{l}\mathrm{E} \\
\mathrm{M}\end{array}$} & $\mathrm{MCHC}$ & $\operatorname{gr} \%$ & 34.2 & $33.5-34.6$ & $31.0-36.0$ \\
\hline & $\mathrm{MCH}$ & pgr & 14.9 & $14.1-17.1$ & $12.3-16.2$ \\
\hline \multirow{2}{*}{$\begin{array}{l}\mathrm{A} \\
\mathrm{T}\end{array}$} & MPV & $\mathrm{fl}$ & 18.9 & $14.2-34.7$ & $8.0-26.0$ \\
\hline & Platelets & $\mathrm{mm}^{3}$ & 278,500 & $106,000-437,000$ & $150,000-500,000$ \\
\hline \multirow{2}{*}{$\begin{array}{l}\mathrm{O} \\
\mathrm{L}\end{array}$} & RDW & $\%$ & 15.7 & $13.8-17.9$ & $13.0-17.0$ \\
\hline & WBC & $/ \mathrm{mm}^{3}$ & 20,470 & $8,050-46,220$ & $4,800-14,930$ \\
\hline \multirow{6}{*}{$\begin{array}{l}\mathrm{O} \\
\mathrm{G} \\
\mathrm{Y}\end{array}$} & Neutrophils & $/ \mathrm{mm}^{3}$ & 10,885 & $3,689-34,440$ & $1,600-10,000$ \\
\hline & Band neutrophils & $/ \mathrm{mm}^{3}$ & 2,105 & $0-32,340$ & 0 \\
\hline & Basophils & $/ \mathrm{mm}^{3}$ & 0 & $0-180$ & $0-10$ \\
\hline & Eosinophils & $/ \mathrm{mm}^{3}$ & 164 & $0-770$ & $60-1470$ \\
\hline & Lymphocytes & $/ \mathrm{mm}^{3}$ & 1,173 & $462-3,181$ & $900-5600$ \\
\hline & Monocytes & $/ \mathrm{mm}^{3}$ & 135 & $0-1,128$ & $0-650$ \\
\hline
\end{tabular}

$\mathrm{RCBs}=$ Red blood cell count $\mathrm{MCV}=$ Mean corpuscular volume; $\mathrm{MCHC}=$ Mean corpuscular hemoglobin concentration; $\mathrm{MCH}=$ Mean corpuscular hemoglobin; $\mathrm{MPV}=$ Mean platelet volume; $\mathrm{RDW}=$ Red cell distribution width; $\mathrm{WBC}=$ White blood cell count.

10 cats were successfully treated with medical therapy combining SBTT and antibacterial treatment, whereas in the other $7 / 10$ cats, surgical exploration of the thoracic cavity was performed.

The indications for surgical intervention were the following: the suspected presence of a foreign body (3/10), pulmonary abscess $(2 / 10)$, mediastinal granulomas $(1 / 10)$ at diagnostic imaging, and the persistence and worsening of septic effusion unresponsive to thoracic drainage and antimicrobial treatment $(1 / 10)$. The surgical approach was chosen based on diagnostic imaging results and varied between sternotomy (4/7) and intercostal thoracotomy (3/7). During surgical exploration of the thorax, the following were found: a mediastinal granuloma (1/7), plant foreign bodies (3/7), pulmonary abscesses $(2 / 7)$, and persistent septic exudate and abscesses in the pleural space (1/7) (Table 1). In all cases, after the surgical removal of the lesion, extensive thoracic irrigation with warm sterile $\mathrm{NaCl} 0.9 \%$ was performed, and the SBTTs were reinserted. Histopathology confirmed a final diagnosis of either abscess or granuloma for cats showing pulmonary and mediastinal lesions at surgical exploration, respectively.

The median time of permanence of the SBTT after surgery was 4.5 days (range: 3-12 days). In 1/7 cat, complete occlusion of the SBTT occurred in the postoperative period, necessitating drain removal.

All the cats were discharged from hospital after a median of 8.5 days (range: 6-18 days). In particular, the cats undergoing surgery were sent home after a median time of 5 days after surgery (range: 4-10 days) and were hospitalized for a total median time of 10 days (range: 6-18 days), while the cats treated conservatively were hospitalized for a median duration of 7 days (range: 6-15 days).

\section{Discussion}

LBTTs have traditionally been advocated in the treatment of pyothorax in small animals because it is believed they could provide greater performance in terms of the amount of fluid and air drained out (Barrs and Beatty, 2009b). In this regard, the management of pyothorax represents a challenge due to the thickness of the exudate, especially in the chronic stages, with subsequent failure of the drain. Nonetheless, SBTTs have recently been suggested to be an effective alternative to LBTTs for the management of pleural space disease, including pyothorax, in both humans and animals (Valtolina and Adamantos, 2009; Hooper et al., 2015b; Filosso et al., 2016; Fetzer et al., 2017; Hallifax et al., 2017). 
Table 3. Biochemistry and blood clotting test at presentation of the 10 cats enrolled in the study.

\begin{tabular}{|c|c|c|c|c|c|}
\hline & Variable & Unit & Median value & $\begin{array}{c}\text { Range } \\
\text { (min-max) }\end{array}$ & Reference interval \\
\hline \multirow[b]{4}{*}{ B } & ALT & $\mathrm{U} / 1$ & 39 & $21-66$ & $20-72$ \\
\hline & AST & $\mathrm{U} / 1$ & 122 & $30-205$ & $9-40$ \\
\hline & GGT & $\mathrm{U} / 1$ & 1.4 & $0.1-5.1$ & $0-4.0$ \\
\hline & SAP & $\mathrm{U} / 1$ & 12 & $2-21$ & $20-140$ \\
\hline I & Creatinine & $\mathrm{mg} / \mathrm{dl}$ & 0.9 & $0.6-1.7$ & $0.8-1.8$ \\
\hline $\mathrm{O}$ & Urea & $\mathrm{mg} / \mathrm{dl}$ & 48 & $30-179$ & $30-65$ \\
\hline \multirow{2}{*}{ C } & Glucose & $\mathrm{mg} / \mathrm{dl}$ & 90 & $41-269$ & $65-148$ \\
\hline & Total protein & $\mathrm{g} / \mathrm{dl}$ & 6.5 & $4.8-8.9$ & $6.5-8.8$ \\
\hline \multirow{2}{*}{$\mathrm{E}$} & Albumin & $\mathrm{g} / \mathrm{dl}$ & 2.2 & $1.7-2.6$ & $2.6-4.0$ \\
\hline & $\mathrm{A} / \mathrm{G}$ ratio & - & 0.48 & $0.35-0.67$ & $0.52-1.20$ \\
\hline M & Bilirubin & $\mathrm{mg} / \mathrm{dl}$ & 0.26 & $0-3.18$ & $0-0.35$ \\
\hline I & $\mathrm{CK}$ & IU/1 & 260 & $139-1048$ & $91-326$ \\
\hline $\mathrm{S}$ & Cholesterol & $\mathrm{mg} / \mathrm{dl}$ & 135 & $113-198$ & $59-230$ \\
\hline $\mathrm{T}$ & SAA & $\mu \mathrm{mol} / 1$ & 157 & $0-198$ & $0-5$ \\
\hline $\mathrm{R}$ & Calcium & $\mathrm{mg} / \mathrm{dl}$ & 8.5 & $8.1-9.0$ & $8.5-10.5$ \\
\hline \multirow[t]{5}{*}{$\mathrm{Y}$} & Correct calcium & $\mathrm{mg} / \mathrm{dl}$ & 9.9 & $8.9-10.0$ & $6.5-10.5$ \\
\hline & Sodium & $\mathrm{mEq} / 1$ & 149 & $143-156$ & $145-155$ \\
\hline & Potassium & $\mathrm{mEq} / \mathrm{l}$ & 4.3 & $3.4-5.0$ & $3.4-5.1$ \\
\hline & Magnesium & $\mathrm{mEq} / \mathrm{dl}$ & 2.5 & $1.9-3.8$ & $1.9-2.8$ \\
\hline & Chloride & $\mathrm{mEq} / \mathrm{dl}$ & 114 & $111-118$ & $110-123$ \\
\hline \multirow[t]{2}{*}{$\mathrm{BCT}$} & PT & Second & 8.1 & $7.3-9.4$ & $9.0-15.0$ \\
\hline & aPTT & Second & 15.0 & $11.5-120.0$ & $9.0-20.0$ \\
\hline
\end{tabular}

$\mathrm{ALT}=$ Alanine aminotransferase $; \mathrm{AST}=$ Aspartate aminotransferase $; \mathrm{GGT}=$ Gamma glutamyl transferase $; \mathrm{SAP}=$ Serum acid phosphatase; $\mathrm{A} / \mathrm{G}$ ratio $=$ Albumin to globulin ratio; $\mathrm{CK}=$ Creatine phosphokinase; $\mathrm{SAA}=$ Serum amyloid $\mathrm{A}$; $\mathrm{BCT}=$ Blood clotting tests; $\mathrm{PT}=$ Prothrombin time; aPTT = Activated partial thrombin time .

Table 4. Hemogas analysis at presentation of the 10 cats enrolled in the study.

\begin{tabular}{cccccc}
\hline & Variable & Unit & Median value & $\begin{array}{c}\text { Range } \\
\text { (min-max) }\end{array}$ & Reference interval \\
\hline $\mathrm{H}$ & $p H$ & - & 7.28 & $7.02-7.39$ & $7.24-7.37$ \\
$\mathrm{~A}$ & $\mathrm{pCO}_{2}$ & $\mathrm{mmHg}$ & 40.4 & $11.4-53.9$ & $31.3-46.3$ \\
$\mathrm{E}$ & $\mathrm{HCO}_{3}^{-}$ & $\mathrm{mmol} / 1$ & 17.7 & $10.8-22.2$ & $15.3-21.2$ \\
$\mathrm{M}$ & Anion gap & $\mathrm{mmol} / 1$ & 11.6 & $0.6-20.0$ & $9.3-18.3$ \\
$\mathrm{O}$ & Base excess & $\mathrm{mmol} / 1$ & -6.4 & $-18.0-0$ & $0 \pm 4.0$ \\
$\mathrm{G}$ & Sodium & $\mathrm{mmol} / 1$ & 145 & $136-152$ & $147-154$ \\
$\mathrm{~A}$ & Ionized calcium & $\mathrm{mmol} / 1$ & 1.18 & $1.03-1.29$ & $1.19-1.39$ \\
$\mathrm{~S}$ & Chloride & $\mathrm{mmol} / 1$ & 123 & $109-131$ & $118-128$ \\
\hline
\end{tabular}

$\mathrm{pCO}_{2}=$ Partial pressure of carbon dioxide.

In the present study, the insertion of SBTTs was safe with only one major complication (malpositioning requiring repositioning) registered. The incidence of pneumothorax was lower as compared to the study of
Valtolina and Adamantos (2009), and the thoracostomy tube placed permitted draining the air and treating this complication. During the permanence of the SBTT, only one major complication was registered which was 
the complete obstruction of the SBTT due to severe pulmonary adhesions and the presence of numerous pockets of purulent material.

Due to the evidence of communication between the two hemithoraces in the presence of severe inflammation (Von Recum, 1977; Epstein, 2014), in the VUH in the study, a conservative approach was chosen, inserting a unilateral drain regardless of the effusion distribution. This approach reduced the number of drains inserted and, consequently, the time of sedation or the need of anesthesia. Thoracic ultrasound was used to confirm the efficacy of the unilateral drains for pleural fluid removal. In only $1 / 10$ cats was a second thoracostomy tube inserted the day after the first insertion, while in two cats the surgeon on duty decided to apply bilateral SBTTs during the first procedure. It is possible that the condition of these cats was considered more severe and the drainage after the positioning of the first SBTT was not satisfying.

The SBTT appeared easy to insert, as malpositioning necessitating drain reinsertion occurred in one case only. Furthermore, mild sedation was sufficient for SBTT insertion in the majority of cases $(8 / 10,80 \%)$. The latter data are worth noting, as the use of SBTTs might reduce the need for general anesthesia (Tillison, 1997; Valtolina and Adamantos, 2009) for thoracic tube placement in potentially unstable patients with respiratory distress. On the contrary, the traditional use of LBTTs always requires total anesthesia.

In the case of LBTTs placement, pain control represents the main issue in the post-insertion period, especially in cats. Adequate pain control was achieved with buprenorphine in almost all the cats $(9 / 10$, $90 \%$ ) included in the study, thus indicating SBTTs to be generally well-tolerated devices in the postinsertion period. In fact, the use of methadone, a pure opioid agonist with a more potent analgesic effect as compared with buprenorphine (Robertson and Taylor, 2004), was needed in only $1 / 10$ cats. Moreover, the use of a single drain could also reduce the discomfort in the cats. Unfortunately, due to the retrospective nature of the study, no pain scales could be applied for a more objective evaluation of pain management.

Moreover, SBTTs appeared to be effective in the evacuation of purulent material from the thoracic cavity; although partial obstruction occurred in approximately one-third of the cats, the possibility of evacuating the exudate was generally not compromised. Regular lavages of the drains with warm sterile solutions carried out as standard care in the patients enrolled might have been responsible for the low rate of complete occlusion reported. Although no consensus regarding the usefulness of thoracic lavage can be found in the veterinary literature, their beneficial role has been suggested by Valtolina and Adamantos (2009), who have stated that this type of management is sufficient to avoid blockage of the drain; this has also been suggested by other studies (Padrid, 2000; Bjorling, 2001; Barrs et al., 2005; Barrs and Beatty, 2009b; Boothe et al., 2010). There is no known advantage in carrying out thoracic cavity irrigation in dogs or cats with anything other than sterile physiologic solutions (MacPhail, 2007). In this regard, regular thoracic lavages of the drains have been seen to be beneficial and have been associated with better outcomes in both people with pleural infection (Hooper et al., 2015a), and in dogs and cats with pyothorax (Demetriou et al., 2002). In only one case was the SBTT unable to drain because of severe pleural adhesions which created purulent cavities filled with a large amount of flocculent effusion requiring surgery. Moreover, in one case in the postoperative period, the SBTT was unable to drain the fluid present and was removed without replacement; however, the cat recovered uneventfully.

Criteria for deciding whether conservative management of pyothorax has failed have not yet been established in the veterinary literature. In this regard, some authors have recommended that, if there is no improvement within 3-7 days or if there is clinical deterioration in spite of the medical therapy, more aggressive methods of treatment are required (Barrs and Beatty, 2009b). Recent studies have shown the benefit of surgical intervention: surgical treatment was more likely to be successful than medical therapy in dogs (Rooney and Monnet, 2002), as well as in cats, as those who received surgical intervention had a high survival rate (Waddell et al., 2002). In the current case series, surgical treatment was carried out in the majority of the cats (7/10 cats), and the prevalence of surgery was higher than that already reported in previous studies (Valtolina and Adamantos, 2009; Epstein, 2014). These data may have been influenced by the surgical assessment criteria applied by the surgeon on duty. In fact, the cats which underwent surgery had documented radiographic pulmonary or mediastinal lesions for which medical management was not deemed appropriate; therefore, the need for surgical exploration was not considered linked to the inability of the SBTT to drain the purulent exudate. However, it was not possible to exclude the fact that the performance of the SBTT could have been linked to the large incidence of surgery in the cats included in the study.

Feline pyothorax generally has a favorable prognosis, with survival rates ranging from $62 \%$ to $100 \%$ depending on the type of treatment selected and the studies considered (MacPhail, 2007; Crawford et al., 2011; Epstein, 2014). The insertion of SBTT gave proof of being a valid option in the emergency phase to stabilize the patient, due to the lower risks linked to its insertion and the effective capability of draining purulent exudate. All the cats included in the current study survived to hospital discharge. It could be suggested that the initial use of SBTTs, combined with surgical treatment in the case of no improvement or lesions requiring surgery, was responsible for the successful management of the cases presented. This 
assumption, however, remains speculative as the study was limited by the low number of cats included and by its retrospective nature. In particular, some complications may not have been reported, especially minor ones.

In conclusion, SBTT represents a valid and safe option for the management of feline pyothorax, having a lower rate of complications when compared to previously reported results. Moreover, the SBTTs appeared to be both easy to insert, allowing the use of short sedation protocols for their insertion, and to be well tolerated by the feline patient once in place. Large-scale prospective studies are necessary in order to compare SBTTs to LBTTs for the treatment of feline pyothorax, and to postulate definite guidelines for managing the disease.

\section{Conflict of interest}

The authors declare that there is no conflict of interest. The authors received no financial support for the research, authorship, and/or publication of this article.

\section{References}

Barrs, V.R. and Beatty, J.A. 2009a. Feline pyothorax - new insights into an old problem: part 1. Aetiopathogenesis and diagnostic investigation. Vet. J. 179, 163-170.

Barrs, V.R. and Beatty, J.A. 2009b. Feline Pyothoraxnew insights into an old problem: part 2. Treatment recommendations and prophylaxis. Vet. J. 179, 171-178.

Barrs, V.R., Allan, G.S., Martin, P., Beatty, J.A. and Malik, R. 2005. Feline pyothorax: a retrospective study of 27 cases in Australia. J. Feline Med. Surg. 7, 211-222.

Beatty, J. and Barrs, V. 2010. Pleural effusion in the cat: a practical approach to determining aetiology. J. Feline Med. Surg. 12, 693-707.

Bjorling, D.E. Management of pyothorax: a medical or surgical disease? In the Proceedings of the 44th British Small Animal Veterinary Association Congress, Birmingham, UK, 2001, pp 58-60.

Boothe, H.W., Howe, L.M., Boothe, D.M., Reynolds, L.A. and Carpenter, M. 2010. Evaluation of outcomes in dogs treated for pyothorax: 46 cases (1983-2001). J. Am. Vet. Med. Assoc. 236, 657663.

Cola, V., Del Magno, S., Valentini, S., Zanardi, S., Foglia, A., Spinella, G., Capitani, O., Buracco, P. and Pisoni, L. 2019. Deep vegetal foreign bodies in cats: a retrospective study of 10 cases. J. Am. Anim. Hosp. Assoc. 55, 249-255.

Crawford, A.H., Halfacree, Z.J., Lee, K.C. and Brockman, D.J. 2011. Clinical outcome following pneumonectomy for management of chronic pyothorax in four cats. J. Feline Med. Surg. 13, 762-767.

Demetriou, J.L., Foale, R.D., Ladlow, J., McGrotty, Y., Faulkner, J. and Kirby, B.M. 2002. Canine and feline pyothorax: a retrospective study of 50 cases in the UK and Ireland. J. Small Anim. Pract. 43, 388-394.

Drobatz, K.J., Hopper, K., Rozanski, E.A. and Silverstein, D.C. 2018. Textbook of small animal emergency medicine. Hoboken, NJ: John Wiley \& Sons.

Epstein, S.E. 2014. Exudative pleural diseases in small animals. Vet. Clin. North Am. Small Anim. Pract. 44, 161-180.

Fetzer, T.J., Walker, J.M. and Bach, J.F. 2017. Comparison of the efficacy of small and large-bore thoracostomy tubes for pleural space evacuation in canine cadavers. J. Vet. Emerg. Crit. Care 27, 301-306.

Filosso, P.L., Sandri, A., Gurrera, F., Ferraris, A., Marchisio, F., Bora, G., Costardi, L., Solidoro, P., Ruffini, E. and Oliaro A. 2016. When size matters: changing opinion in the management of pleural space - the rise of small-bore pleural catheters. J. Thorac. Dis. 8, 503-510.

Hallifax, R.J., Psallidas, I. and Rahman, N.M. 2017. Chest drain size: the debate continues. Curr. Pulmonol. Rep. 6, 26-29.

Hooper, C.E., Edey, A.J., Wallis, A., Clive, A.O., Morley, A., White, P., Medford, A.R.L., Harvey, J.E., Darby, M., Zahan-Evans, N. and Maskell, N.A. 2015a. Pleural irrigation trial (PIT): a randomized controlled trial of pleural irrigation with normal saline versus standard care in patients with pleural infection. Eur. Respir. J. 46, 456-463.

Hooper, C.E., Welhalm, S.A. and Maskell, N.A. 2015 b. Pleural procedures and patient safety: a national BTS audit of practice. Thorax. 70, 189-191.

MacPhail, C.M. 2007. Medical and surgical management of pyothorax. Vet. Clin. North Am. Small Anim. Pract. 37, 975-988.

Padrid, P. 2000. Canine and feline pleural disease. Vet. Clin. North Am. Small Anim. Pract. 30, 1295-1307.

Robertson, S.A. and Taylor, P.M. 2004. Pain management in cats: past, present and future. Part 2. Treatment of pain-clinical pharmacology. J. Feline Med. Surg. 6, 321-333.

Rooney, M.B. and Monnet, E. 2002. Medical and surgical treatment of pyothorax in dogs: 26 cases (1991-2001). J. Am. Vet. Med. Assoc. 221, 86-92.

Sherman, A., Holt, D., Drobatz, K. and Mison, M. 2020. Evaluation of Jackson-Pratt thoracostomy drains compared with traditional trocar type and guidewire-inserted thoracostomy drains. J. Am. Anim. Hosp. Assoc. 56, 92-97.

Sigrist, N.E. 2015. Thoracostomy tube placement and drainage. In: Small animal critical care medicine, 2nd ed. Eds., Silverstein, D.C. and Hopper, K.E. St. Louis, MO: Elsevier Saunders, pp: 1032-1036.

Stillion, J.R. and Letendre, J. 2015. A clinical review of the pathophysiology, diagnosis, and treatment 
of pyothorax in dogs and cats. J. Vet. Emerg. Crit. Care 25, 113-129.

Tillison, D.M. 1997. Thoracostomy tubes. Part I. Indications and anesthesia. Compend. Contin. Educ. Pract. Vet. 19, 1258-1263.

Valtolina, C. and Adamantos, S. 2009. Evaluation of small-bore wire-guided chest drains for management of pleural space disease. J. Small Anim. Pract. 50, 290-297.
Von Recum, A.F. 1977. The mediastinum and haemothorax, pyothorax and pneumothorax in the dog. J. Am. Vet. Med. Assoc. 171, 531-533.

Waddell, L.S., Brady, C.A. and Drobatz, K.J. 2002. Risk factors, prognostic indicators, and outcome of pyothorax in cats: 80 cases (1986-1999). J. Am. Vet. Med. Assoc. 221, 819-824. 